\title{
Seeking Medical Support for Depression after the Childbirth: A Study of Japanese Community Mothers of 3-Month-Old Babies
}

\author{
Toshinori Kitamura*,1 $^{*}$ Hiroshi Yamashita ${ }^{2}$ and Keiko Yoshida ${ }^{2}$ \\ ${ }^{I}$ Department of Clinical Behavioural Sciences (Psychological Medicine), Kumamoto University Graduate School of \\ Medical Sciences, Kumamoto, Japan \\ ${ }^{2}$ Department of Neuropsychiatry, Kyushu University Hospital, Japan
}

\begin{abstract}
Mothers of 3-month-old babies ( $\mathrm{N}=1,298)$ were investigated, about 5\% of whom reported depression. Of the women with depression, $7 \%$ visited a medical institution for their condition. The mothers with depression who did not seek medical support scored significantly higher in bonding failure than those who did so. Less frequent spontaneous medical support seeking among mothers with both depression and bonding failure underscores the importance of assertive (outreach) community mental health care for postnatal women.
\end{abstract}

Keywords: Postnatal depression, bonding failure, child abuse, parents' age.

\section{INTRODUCTION}

Depression after the childbirth is a major issue in women's mental health [1]. Despite the high prevalence of women with depression during the postnatal period, qualitative research demonstrates that such women do not proactively seek help. An Icelandic study showed that $5 \%$ of mothers of 2-month-old babies were severely distressed (diagnosis not clear) and that only one out of four subsequently received health care for their condition [2]. Of such women with depression, about $7 \%$ sought medical help. Fujihara and Kitamura [3] reported that about $10 \%$ of people with a depressive illness living in a Japanese community visited a medical institution. However, there has been no report to our knowledge that has assessed how many women seek medical support for their depression after the childbirth in Japan.

Barriers to seeking help include women's inability to disclose their feelings and family members' and health professionals' reluctance to respond to the mothers' emotional and practical needs [4]. These barriers may differ between cultures. Identifying factors that correlate with women's reluctance to seek treatment for their depression after the childbirth may yield insight into the ways health professionals can best provide care in community settings.

Mothers with infants need attention from mental health professionals not only because of postnatal depression but also because of the potential for bonding failure [5,6], a lack of or reduced maternal affection for the baby that causes severe distress among mothers. Also of importance in terms of postnatal mental heath care is abusive parenting. Depressed mothers are more likely to adopt abusive parental styles [7]. It is crucial, then, that mothers with depression, bonding failure, and abusive parenting during the postnatal

*Address correspondence to this author at the Department of Clinical Behavioural Sciences (Psychological Medicine), Kumamoto University Graduate School of Medical Sciences, 1-1-1 Honjo, Kumamoto, Kumamoto, 860-8556, Japan; E-mail kitamura@kumamoto-u.ac.jp period are provided with sufficient mental health care. If depressed mothers seek medical support spontaneously, sufficient institution-based care may be available. If they do not, however, assertive mental health care such as outreach services by community nurses may be necessary.

This study investigates medical support seeking among Japanese community women with depression after the childbirth. We were primarily interested in the prevalence of such support seeking and its correlates. The research questions were; (1) how much proportion of postnatal women experienced depression, (2) how many of such depressed women sought medical support spontaneously, and (3) what were correlated of such medical support seeking among depressed women during the postnatal period.

\section{METHODS}

\section{Participants}

When mothers attended a 3-month postnatal health check-up organised by four cities, 21 towns, and five villages in Kumamoto Prefecture (located in the centre of the Kyushu Island southwest to Tokyo), they were requested to fill in the questionnaire anonymously while waiting for the check-up and to return it prior to leaving. The number of questionnaires distributed was 1,405 , of which $1,293(92 \%)$ were returned.

\section{Instruments}

Due to time restrictions and physical limitations (mothers filled in the questionnaire while holding their babies), the questionnaire was restricted to a single page. The number of items used, therefore, was reduced as much as possible.

Depression during the postnatal period was assessed by a single item: "Have you felt sad or miserable during the last week?", with a 4-point scale from "never" $(0)$ to "almost always" (3). Although this is not a very accurate measure of depression and a scale with multiple items would be more desirable, we believe that this single-item assessment is suf- 
ficient in a community-based study such as our own because the validity of such a single item as a screening instrument for depression has been reported. For example, Chochinov and colleagues [8] applied a single item question of depressed mood extracted from the Schedule for Affective Disorders and Schizophrenia (SADS [9]) among 197 patients receiving palliative care for advanced cancer and found that such question identified depression rated by an structured diagnostic interview (SADS) and the diagnostic criteria (Research Diagnostic Criteria [10]) extremely accurately. Its sensitivity (1.00) and specificity (1.00) were higher than those obtained for a multiple item questionnaire, the Beck Depression Inventory [11]. Reviewing and meta-analysing a single-item and two-item screening instruments of depression, Mitchell and Coyne [12] reported that an overall sensitivity of $32 \%$ and specificity of $97 \%$ of a single-item screen. not all people identified as depressed by such an instrument are truly depressed (positive predictive value 56\%) whereas most of people identified as not depressed by such an instrument are truly non-depressed (negative predictive value 97\%). Thus the estimation of depression in this study may be underestimation.

This was followed by the questionnaire - "Have you visited a medical institution for this?" - requiring a forced answer choice of "Yes" or "No". Details of medical support such as medication or psychotherapy were not investigated.

Bonding failure was evaluated by two items that appear on the Mother-Infant Bonding Questionnaire (MIBQ): "I feel protective towards my baby" and "I feel close to my baby" (reverse items). The MIBQ aims to elicit from mothers their feelings of rejection, alienation, and neutrality towards their infants (Kumar et al. and revised by Marks et al., unpublished). The study that originally presented the MIBQ showed excellent test-retest reliability and construct validity [13]. Its validity has also been reported [14,15]. the scale was translated into Japanese (Yoshida K, Yamashita H, Conroy S, Marks, MN, Kumar R. A mother-to-infant bonding scale: Stability and links with maternal mood in early postnatal period in Japan. in preparation) and used in a clinical study [16].

The presence of an abusive parenting style was determined by asking for the number of occurrences since childbirth of three items extracted from the Child Form of the Conflict Tactic Scale (CTS) [17]: "insulted or swore at him/her", "sulked or refused to talk about an issue", and "cried". We extracted these three items as they scored highest in a previous study on Japanese mothers' child abuse of one-month-old babies [7]. Our analyses used the combined number of these three behaviours.

Regarding demographic and obstetric data, we enquired about the mothers' and their partners' ages as well as the infants' birth weights and gender.

\section{Statistical Analyses}

After examining the frequency of the participants' response to the depression item, we estimated the rate of medical support seeking among depressed mothers. Both depression and medical support seeking were then correlated to the participants' and their partner's ages, weight and gender of the infant, abusive parental style, and bonding failure. For statistical analysis the Statistical Packages for Social Sciences (SPSS) version14.0 was used.

\section{Ethical Consideration}

This research project was approved by the Ethical Committee of Kumamoto University Graduate School of Medical Sciences.

\section{RESULTS}

In response to the question about depression, $12(0.9 \%)$ mothers answered "almost always", 46 (3.6\%) mothers "many times", $276(21.3 \%)$ mothers "few times", and 959 $(74.2 \%)$ mothers "never". The mothers in the first two categories ("almost always" and "many times") were regarded as suffering from depression. Of these mothers, two (16.7\%), two $(4.3 \%)$, zero $(0.0 \%)$, and one $(0.1 \%)$, respectively, reported having visited a medical institution for depression.

Using the medical institution visit as a dummy variable (no visit $=0$, any visit $=1$ ), we calculated correlations with the other variables. The medical institution visit correlated significantly with depression and abusive parental style (Table 1), while depression, abusive parenting style, and bonding failure correlated significantly with each other. Finally, depression and abusive parenting style were significantly associated with younger ages of the mothers and their partners.

Among 58 mothers who experienced depression at least "many" times a week, four (6.9\%) sought medical support. Among the group of 58, the MIBQ score was significantly ( $\mathrm{t}$ $=4.86, p<.001)$ higher among those mothers who did not seek medical support (mean $=1.37, \mathrm{SD}=0.56)$ than among those who did (mean $=1.00, \mathrm{SD}=0.00)$. The CTS scores were lower among those mothers who did not seek medical support (mean $=0.98, \mathrm{SD}=2.48$ ) than among those who did (mean $=2.75, \mathrm{SD}=4.84)$, but this did not reach a statistically significant level $(\mathrm{t}=0.72, p=.520)$.

\section{DISCUSSION}

This study has shown that about $5 \%$ of mothers of 3 month-old babies suffer from depression currently. This figure echoes the findings of an interview-based epidemiological investigation of perinatal mental disorders among primiparae in five university-based clinics in Japan, in that 5\% of women had an onset of DSM-III-R major depressive episode in the 3-month postnatal period [18]. This is also in line with a meta-analysis of the prevalence of postnatal depression [1].

The rate of medical support seeking among the women in this study is slightly lower than that previously reported for the depressed population in Japan [3]. This very low rate among postnatal women with depression poses a serious concern for mental health professionals and administrators because depression during the perinatal period results in not only subjective discomfort in the mothers but also adverse effects on the subsequent development of the child [19-22], mother-child bonding [23] and parenting practice [24].

A unique finding of this study is that among postnatal women with depression, those who did not seek medical support suffered from more severe bonding failure than those who did. Women with bonding failure are more likely to be depressed [23] and women with both depression and bonding 
Table 1. Means and SDs of and Correlations Between all the Variables Used in this Study $(\mathbf{n}=1,293)$

\begin{tabular}{|c|c|c|c|c|c|c|c|c|}
\hline & 1 & 2 & 3 & 4 & 5 & 6 & 7 & 8 \\
\hline 2 depression & $.18^{* * *}$ & --- & & & & & & \\
\hline 3 own age ${ }^{a}$ & .02 & $-.06^{*}$ & --- & & & & & \\
\hline 5 weight of the infant ${ }^{c}$ & .02 & .03 & .04 & .03 & --- & & & \\
\hline 6 gender of the infant ${ }^{d}$ & .04 & -.04 & .03 & .04 & $-.06^{*}$ & --- & & \\
\hline 7 abusive parenting style & $.12 * * *$ & $.22 * * *$ & $-.07 *$ & $-.06^{*}$ & -.03 & -.05 & --- & \\
\hline 8 bonding failure ${ }^{e}$ & -.03 & $.15^{* * *}$ & -.02 & -.06 & .02 & .02 & $.13 * * *$ & --- \\
\hline Mean & 0.004 & 0.31 & 29.3 & 31.3 & 3073 & 1.49 & 0.34 & 1.14 \\
\hline SD & 0.062 & 0.58 & 5.2 & 6.3 & 420 & 0.50 & 1.01 & 0.33 \\
\hline
\end{tabular}

${ }^{*} p<.05 ;{ }^{* *} p<.01 ; * * * p<.001 ;{ }^{\mathrm{a}}$ missing values for 14 cases; ${ }^{\mathrm{b}}$ missing values for 23 cases; ${ }^{\mathrm{c}}$ missing values for 47 cases; ${ }^{\mathrm{d}}$ missing values for 21 cases; ${ }^{\mathrm{e}}$ missing values for 11 cases.

failure may be in psychosocial difficulties. Ironically, such mothers are less likely to spontaneously seek support. This emphasises the importance of an assertive community mental health service that should be organised at the level of the local government. Health visitors (community nurses) and midwives could play potentially significant roles in such a service. This is particularly the case in Japan where the stigma of psychiatric diagnosis is enduringly strong $[25,26]$.

Limitations of the present study include the use of a single or a few items for each of the key variables, and the cross-sectional nature of the research design. Thus, mothers who reported having been depressed at the time of the investigation may have begun feeling this way before prior to delivery. Kitamura and colleagues [18] studied 290 Japanese first time mothers and found that 14 women had an onset of Major Depressive Episode for 3-month period after the childbirth whereas nine women were suffering from depression when giving birth. Thus about $60 \%$ of women identified as having depression during the first 3-month period after the childbirth may be suffering from post natal depression. Since about 5\% of the women in this study reported feeling depressed "almost always" or "many times" and this corresponds to the incidence of postnatal depression based on an interview survey, it is likely that these women did in fact suffer from postnatal depression. However this issue awaits future studies that use a structured diagnostic interview.

From the service perspective, mothers with depression need support whether its onset is before or after the delivery. The validity and reliability of the short measures used here need further psychometric verification. However, we used the data at the 3-month postnatal heath check-up because this was legislated in Japan and has been enjoying a very high attendance rate of the postnatal health check-ups. For example, it is $94 \%$ in 2006 for Kumamoto Prefecture [27]. Our use of a large study population residing in a wide area of a
Japanese prefecture and our simultaneous assessment of key variables may warrant a future replication study.

\section{REFERENCES}

[1] O'Hara MW, Swain AM. Rates and risk of postpartum depression: a meta-analysis. Int Rev Psychiatry 1996; 8: 37.

[2] Thome M. Severe postpartum distress in Icelandic mothers with difficult infants: a follow-up study on their health care. Scand J Caring Sci 2003; 17: 104.

[3] Fujihara S, Kitamura T. An epidemiology of mental disorders in an area in Kofu. Nihon Iji Shinpo 1993; 3618: 47. (in Japanese)

[4] Dennis C-L, Chung-Lee L. Postpartum depression help-seeking barriers and maternal treatment preferences: a qualitative systematic review. Birth 2006; 33: 323.

[5] Brockington IF. Diagnosis and management of post-partum disorders: a review. World Psychiatry 2004; 3: 89.

[6] Kumar RC. "Anybody's child": severe disorders of mother-toinfant bonding. Br J Psychiatry 1997; 171: 175.

[7] Kitamura T, Takauma F, Tada K, Yoshida K, Nakano H. Postnatal depression, social support, and child abuse. World Psychiatry 2004; 3: 100 .

[8] Chochinov HM, Wilson KG, Enns M, Lander S. "Are you depressed?": screening for depression in the terminally ill. Am J Psychiatry 1997; 154: 674.

[9] Endicott J, Spitzer RL. A diagnostic interview: the schedule for affective disorders and schizophrenia. Arch Gen Psychiatry 1978; 35: 837 .

[10] Spitzer RL, Endicott J, Robins E. Research diagnostic criteria: rationale and reliability. Arch Gen Psychiatry 1978; 35: 773.

[11] Beck AT, Beck RW. Screening depressed patients in family practice: a rapid technic. Postgrad Med 1972; 52: 81.

[12] Mitchell AJ, Coyne JC. Do ultra-short screening instruments accurately detect depression in primary care? A pooled analysis and meta-analysis of 22 studies. Br J Gen Pract 2007; 57: 144.

[13] Taylor A, Atkins R, Kumar R, Adams D, Glover V. A new motherinfant bonding scale: links with early maternal mood. Arch Women Ment Health 2005; 8: 45.

[14] Wittkowski A, Wiek A, Mann S. An evaluation of two bonding questionnaires: a comparison of the mother-to-infant bonding scale with the postpartum bonding questionnaire in a sample of primiparous mothers. Arch Womens Ment Health 2007; 10: 171.

[15] Figueiredo B, Costa R, Pacheco A, Pais A. Mother-to-infant emotional involvement at birth. Matern Child Health J 2009; doi 10.1107/s10995-0008-0312-X 
[16] Yamashita H, Yoshida K. Investigation of community-based preventive intervention using questionnaires for mothers at risk for child abuse: contribution of perinatal psychiatry to child abuse in infancy. Jpn J Child Abuse Negl 2004; 6: 218. (in Japanese)

[17] Straus MA. Measuring intrafamilial conflict and violence: conflict tactics scales. J Marriage Fam 1979; 41: 75.

[18] Kitamura T, Yoshida K, Okano T, et al. Multicentre prospective study of perinatal depression in Japan: incidence and correlates. Arch Women Ment Health 2006; 9: 121.

[19] Cornish AM, McMahon CA, Ungerer JA, Barnett B, Kowalenko N, Tennant C. Postnatal depression and infant cognitive and motor development in the second postnatal year: the impact of depression chronicity and infant gender. Infant Behav Dev 2005; $28: 407$.

[20] Grace SL, Evindar A, Stewart DE. The effect of postpartum depression on child cognitive development and behavior: A review and critical analysis of the literature. Arch Women Ment Health 2003; 6: 263.

[21] Murray L, Cooper PJ. Eds. Postpartum depression and child development. New York: Guilford Press 1997.

[22] Nadel J, Soussignan R, Canet P, Libert G, Gérardin P. Two-monthold infants of depressed mothers show mild, delayed and persistent change in emotional state after non-contingent interaction. Infant Behav Dev 2005; 28: 418.

[23] Moehler E, Brunner R, Wiebel A, Reck C, Resch F. Maternal depressive symptoms in the postnatal period are associated with longterm impairment of mother-child bonding. Arch Women Ment Health 2006; 9: 273.

[24] Leiferman JA, Ollendick TH, Kunkel D, Christie IC. Mothers' mental distress and parenting practices with infants and toddlers. Arch Women Ment Health 2005; 8: 243.

[25] Hasui C, Sakamoto S, Sugiura T, Kitamura T. Stigmatization of mental illness in Japan: Images and frequency of encounters with diagnostic categories of mental illness among medical and nonmedical university students. J Psychiatry Law 2000; 28: 253.

[26] Sugiura T, Sakamoto S, Tanaka E, Tomoda A, Kitamura T. Stigmatizing perception of mental illness in Japanese students: Comparison of nine psychiatric disorders. J Nerv Ment Dis 2000; 188 : 239.

[27] Kumamoto Prefecture Department of Health Promotion (2007) Kumamoto-ken no boshi hoken Heisei 19 nendo. Mother Child Health in Kumamoto Prefecture 2006.

(C) Kitamura et al.; Licensee Bentham Open.

This is an open access article licensed under the terms of the Creative Commons Attribution Non-Commercial License (http://creativecommons.org/licenses/by-nc/3.0/) which permits unrestricted, non-commercial use, distribution and reproduction in any medium, provided the work is properly cited. 